\title{
Comprehensive Middle-Down Mass Spectrometry Characterization of an Antibody-Drug Conjugate by Combined Ion Activation Methods
}

\author{
Eleanor Watts, ${ }^{1}$ Jon D. Williams, ${ }^{2}$ Laura J. Miesbauer, ${ }^{2}$ Milan Bruncko, ${ }^{2}$ Jennifer S. Brodbelt ${ }^{1 *}$ \\ ${ }^{1}$ Department of Chemistry, University of Texas at Austin, Austin, TX \\ ${ }^{2}$ AbbVie, North Chicago, IL
}

Correspondence to: jbrodbelt@cm.utexas.edu

\section{Supporting Information}

\begin{tabular}{|c|c|c|}
\hline \multicolumn{2}{|l|}{ Content } & \multirow{2}{*}{$\begin{array}{l}\text { Page no. } \\
\text { S2-5 }\end{array}$} \\
\hline Text & Optimization of Activation Parameters & \\
\hline Figure S1. & $\begin{array}{l}\text { Laser energy optimization for UVPD and activation time optimization for ETD and } \\
\text { EThcD. }\end{array}$ & S3 \\
\hline Figure S2. & Precursor isolation window optimization for ETD, EThcD and UVPD. & S4 \\
\hline Figure S3. & Charge state optimization for ETD, EThcD and UVPD. & S5 \\
\hline Figure S4. & MS1 and MS/MS spectra of one payload-containing peptide. & S6 \\
\hline Figure S5. & MS1 and MS/MS spectra of a second payload-containing peptide. & S7 \\
\hline Figure S6. & MS/MS spectra for linker fragmentation with UVPD and HCD & S8 \\
\hline Figure S7. & MS1 spectra for each the seven ADC subunits. & S9 \\
\hline Figure S8. & $\begin{array}{l}\text { Product ion arrays showing sites of backbone cleavages for } \mathrm{Fd}^{\prime} \text { and } \mathrm{Fd}^{\prime} \text { with one } \\
\text { payload }\end{array}$ & S10 \\
\hline Figure S9. & $\begin{array}{l}\text { Product ion arrays showing sites of backbone cleavages for Fd' with two and three } \\
\text { payloads }\end{array}$ & S11 \\
\hline Figure S10. & MS/MS data for EThcD of Fd' with one payload & S12 \\
\hline Figure S11. & MS/MS data for UVPD of Fd' with two payloads & S13 \\
\hline Figure S12. & MS/MS data for ETD of Fd' with two payloads & S14 \\
\hline Figure S13. & MS/MS data for EThcD of Fd' with two payloads & S15 \\
\hline Figure S14. & MS/MS data for UVPD, ETD and EThcD of Fd' with three payloads & S16 \\
\hline Figure S15 & LC trace with percent contributions for LC and Fd' & S17 \\
\hline Table S1. & List of all identified fragment ions for each of the seven ADC subunits & Excel file \\
\hline
\end{tabular}


Optimization of activation parameters. Characterization of payload-containing subunits present additional challenges, thus requiring careful optimization of activation conditions to maximize coverage and bracket modified sites. Owing to gaps in sequence coverage by any single MS/MS method, it was anticipated that the results from multiple activation methods could be combined to afford a higher level of characterization (a strategy also commonly employed for the analysis of antibodies). The experimental parameters of greatest impact include the laser energy and number of laser pulses for UVPD, the ETD activation time, the HCD collision energy, and the width of the mass isolation of the precursor ion. The influence of the charge state of the precursor ion was also evaluated. Each of the activation methods was performed throughout separate LC runs, during which the masses of the payload-conjugated subunits and unmodified subunits were targeted for isolation and activation at prescribed times based on the elution profile displayed in Figure S1-3. All figures display a representative standard deviation for one set of conditions in order to give an idea of the variability.

While the data for the final study was collected in full profile mode and analyzed in TDValidator, optimization data was collected in reduced profile and a streamlined data analysis method was employed in order to allow efficient data analysis using Biopharma Finder. For Biopharma Finder data analysis, a signal-to-noise ratio of 3 and a maximum ppm error of 5 were employed. These standards are typical for reduced profile data which results in lower levels of noise (and less spectral detail) than full profile data.

Figure S1A displays the impact of laser energy on the sequence coverage. There is not a dramatic change in the outcome of UVPD using one laser pulse versus two pulses in Figure S1A. Using a single pulse resulted in a slightly lower dependency on the specific laser energy, especially between 0.25-1.5 mJ. The rationale for this is that when multiple pulses are applied the effects of any variation in laser energy are compounded. In addition, while $0.25 \mathrm{~mJ}$ seemed to provide the highest sequence coverage using two laser pulses, slightly higher laser energy (0.50-1.0 mJ) offered better results when using a single laser pulse. Owing to these comparisons, for the remainder of the study a single pulse was used for UVPD because of the higher degree of reproducibility found in the optimization data and the general trend towards higher sequence coverages observed for each subunit. UVPD using 0.25-1.0 $\mathrm{mJ}$ yielded similar degrees of sequence coverage; however, increasing the energy per laser pulse beyond $1.0 \mathrm{~mJ}$ caused a loss of sequence coverage. This net decrease in sequence coverage is attributed to the prevalence of secondary dissociation and/or excessive energy deposition that contributes to formation of smaller and internal fragment ions, often ones that are redundant or unassignable. Based on the trends in Figure S1A, a laser energy of $1.0 \mathrm{~mJ}$ was selected. Following a similar strategy, the ETD activation time was optimized for ETD and EThCD. Variation of the ETD activation time resulted in relatively modest gains or losses of sequence coverage ( $+5 \%$ relative to the mean coverage) as shown in Figure S1B,C. In order to balance the potential to generate more fragments by using longer activation times with the need for collecting as many spectra as possible to allow averaging for the full profile data analysis, an ETD time of $15 \mathrm{~ms}$ and an EThcD time of $10 \mathrm{~ms}$ were selected.

Figures S2 and S3 illustrate the optimization of precursor charge state and isolation window. There are a few minor differences between the parameters used to initially optimize precursor charge state and isolation window compared to the finalized parameters. First, based on initial examination of Figure S1 a laser energy of $0.5 \mathrm{~mJ}$ per pulse was selected in order to maximize sequence coverage for UVPD spectra acquired using either one or two pulses. After selection of one laser pulse, a final laser energy of $1.0 \mathrm{~mJ}$ was chosen owing to most consistent performance. Similarly, initially an ETD activation time of 30 ms was utilized for optimization of the size of the isolation window and precursor charge state in order to enhance the production of complementary fragment ions for ETD and EThcD (10 ms, $10 \mathrm{NCE}$ ). After extensive comparisons, it was determined that using an activation time that yielded the highest sequence coverage for ETD offered the best comparison to the 
complementary EThCD data. Because the results for charge state and isolation window were generally consistent across activation methods, further re-evaluation of these parameters was not undertaken.

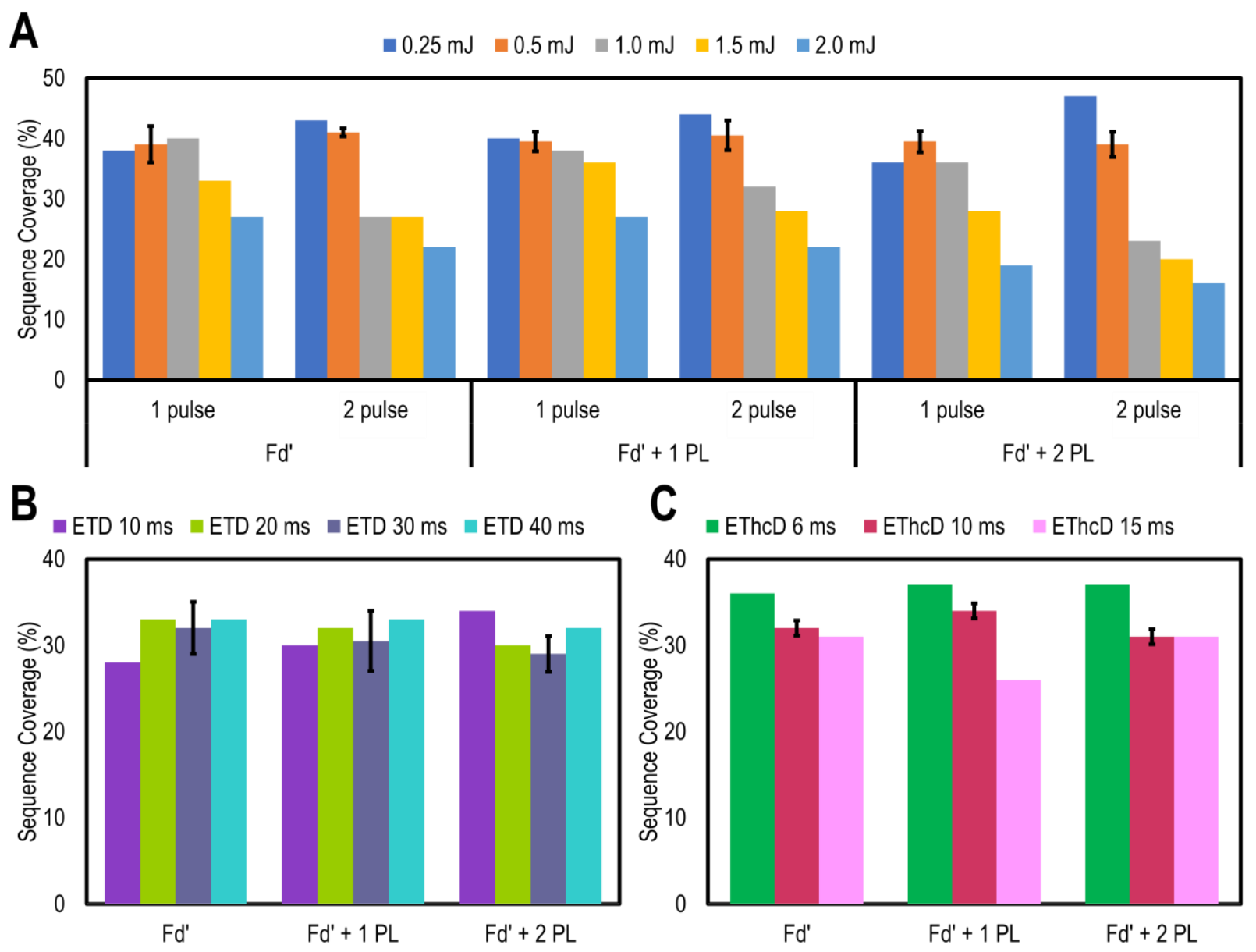

Figure S1: Optimization of activation conditions for (A) UVPD (1 pulse): variation of laser energy, (B) ETD: variation of activation time, and (C) EThcD (HCD energy $10 \mathrm{NCE}$ ): variation of activation time. For this set of comparisons, the $25+$ charge state was selected with an isolation window of $10 \mathrm{~m} / \mathrm{z}$. Results are displayed with the payload (PL) located at C224 for Fd' +1 PL and C230 and C233 for Fd' +2 PL. The typical level of standard deviation is shown for one set of conditions for each activation mode. Error bars represent a $95 \%$ confidence interval based on four replicates. 
The precursor isolation width was also optimized as displayed in Figure $\mathbf{S 2}$ for UVPD (1 pulse, $0.5 \mathrm{~mJ}$ ), ETD (30 ms) and EThcD (10 ms, NCE 10) for the three targeted Fd' species. While other studies have demonstrated improved sequence coverage for ETD and UVPD of antibody subunits using broad isolation windows, ${ }^{1,2}$ decreased sequence coverage was observed when broad isolation windows $(300 \mathrm{~m} / \mathrm{z}$ or 600 $\mathrm{m} / \mathrm{z}$ ) were applied for the ADC subunits in the present study. The loss of sequence coverage is rationalized by considering the features of the MS/MS methods. UVPD produces a large variety of product ions. The fragmentation patterns become overly dense upon activation of multiple co-isolated charge states, resulting in overlapping isotopic envelopes of the ions and impeding ion assignments. For ETD and EThcD, co-isolation and reaction of multiple charge states results in rapid depletion of the ETD reagent ions and generation of product ions in numerous charge states, resulting in lower $\mathrm{S} / \mathrm{N}$ and loss of sequence coverage. Based on these results, individual charge states were isolated and activated for the remainder of the study.

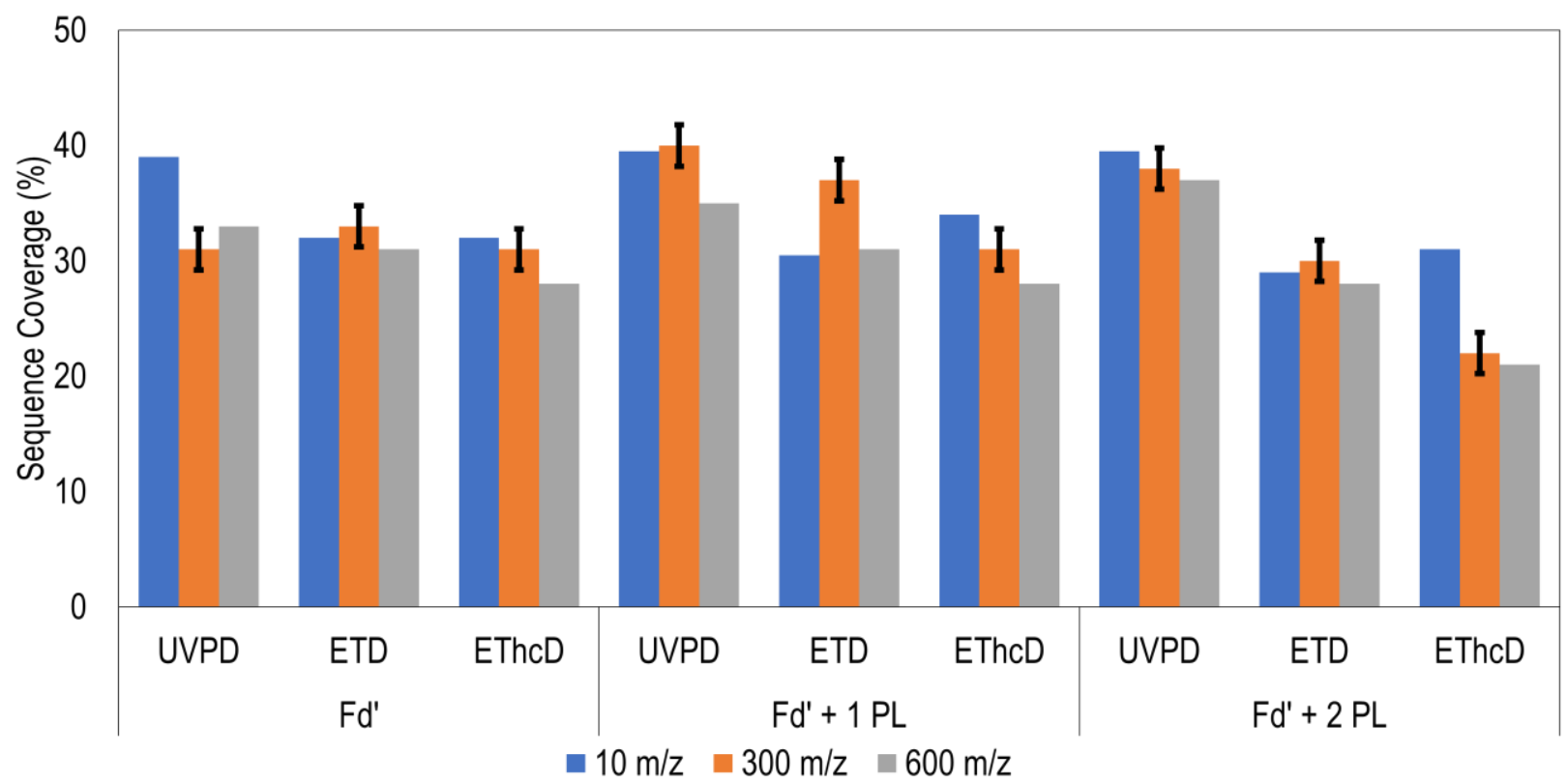

Figure S2: Optimization of precursor isolation window. The reaction time was set to $30 \mathrm{~ms}$ for ETD and $10 \mathrm{~ms}$ for EThcD (NCE 10). For UVPD one $0.5 \mathrm{~mJ}$ pulse was applied. The 25+ charge state was selected for this set of comparisons. Results are displayed with the payload (PL) located at C224 for Fd' +1 PL and C230 and C233 for Fd' +2 PL. The typical level of standard deviation is shown for one set of conditions for each activation mode. Error bars represent a 95\% confidence interval based on four replicates. 
Previous reports have established that the performance of UVPD is not particularly charge state dependent, ${ }^{3,4}$ and the same result is echoed in the present study as illustrated in Figure S3. For this assessment, the sequence coverage obtained for UVPD, ETD and EThcD was measured for three prominent charge states. For all three Fd' species in Figure S3, slightly higher sequence coverage was obtained for the $25+$ charge state, a result attributed to the greater abundance of this charge state which translates to somewhat better S/N of the MS/MS spectra. The efficacy of ETD is known to be charge state dependent, favoring precursors in higher charge states (higher charge density) owing to the greater exothermicity of the electron transfer reactions and reduction of stabilizing non-covalent interactions of the precursor. ${ }^{5,6}$ However, as reported in Figure S3, a significant charge state dependence on sequence coverage was not observed for ETD nor EThcD. Instead, the sequence coverage was generally found to be slightly greater for ETD and EThcD of the $25+$ (mid-range) charge state for all three targeted Fd' species. This outcome which seems to contradict conventional findings about the charge state dependence of ETD is rationalized by the impact of precursor ion abundance. For MS/MS analysis of ADC subunits, a factor that is more important than charge state in modulating sequence coverage is the abundance of the precursor ion. Based on these findings, the charge state of greatest abundance was selected for ETD and EThcD throughout the remainder of the study. Moreover, to obtain maximum coverage, the final method adopted in this study combined the results of three targeted LC runs, each utilizing a different optimized complementary MS/MS strategy (ETD (15 ms), EThcD (10 ms and NCE 10), UVPD (one pulse, $1 \mathrm{~mJ}$ )

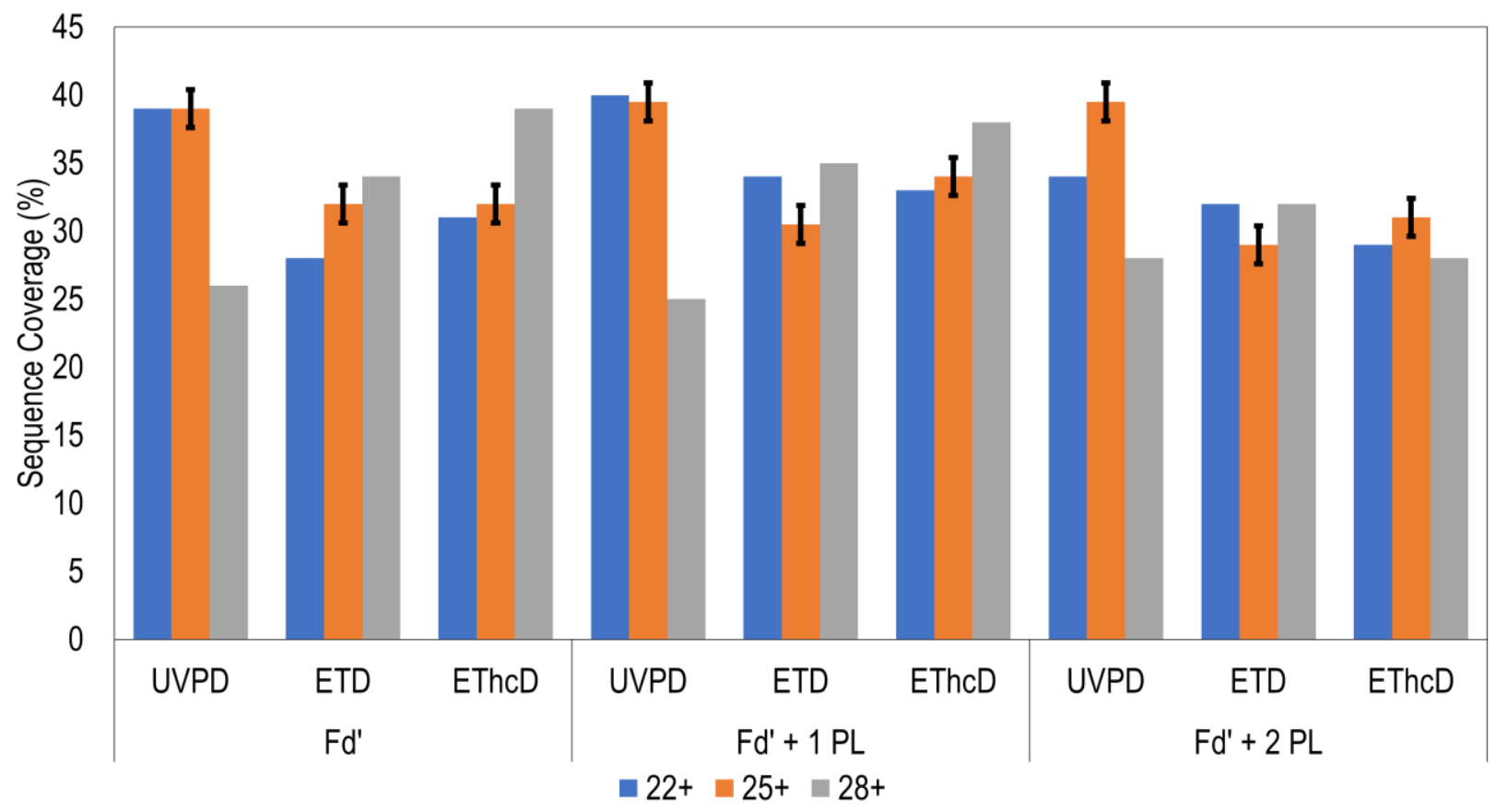

Figure S3: Optimization of charge state. The reaction time was set to $30 \mathrm{~ms}$ for ETD and $10 \mathrm{~ms}$ for EThcD (NCE 10). For UVPD one $0.5 \mathrm{~mJ}$ pulse was applied. For this comparison, the isolation window was set to $10 \mathrm{~m} / \mathrm{z}$. Results are displayed with the payload (PL) located at C224 for Fd' $+1 \mathrm{PL}$ and C230 and C233 for Fd' +2 PL. The typical level of standard deviation is shown for one set of conditions for each activation mode. Error bars represent a 95\% confidence interval based on four replicates. 
Figure S4: (A) MS1 and (B) MS/MS spectra (HCD NCE 30, 4+ charge state) of one payload-containing peptide identified from the Byonic database search of the bottom-up dataset. The spectra are shown for a peptide which is believed to be a correct match. (C) The resulting fragmentation map; no payloadcontaining product ions were identified. The cysteine highlighted in gold is presumed to contain the payload conjugation
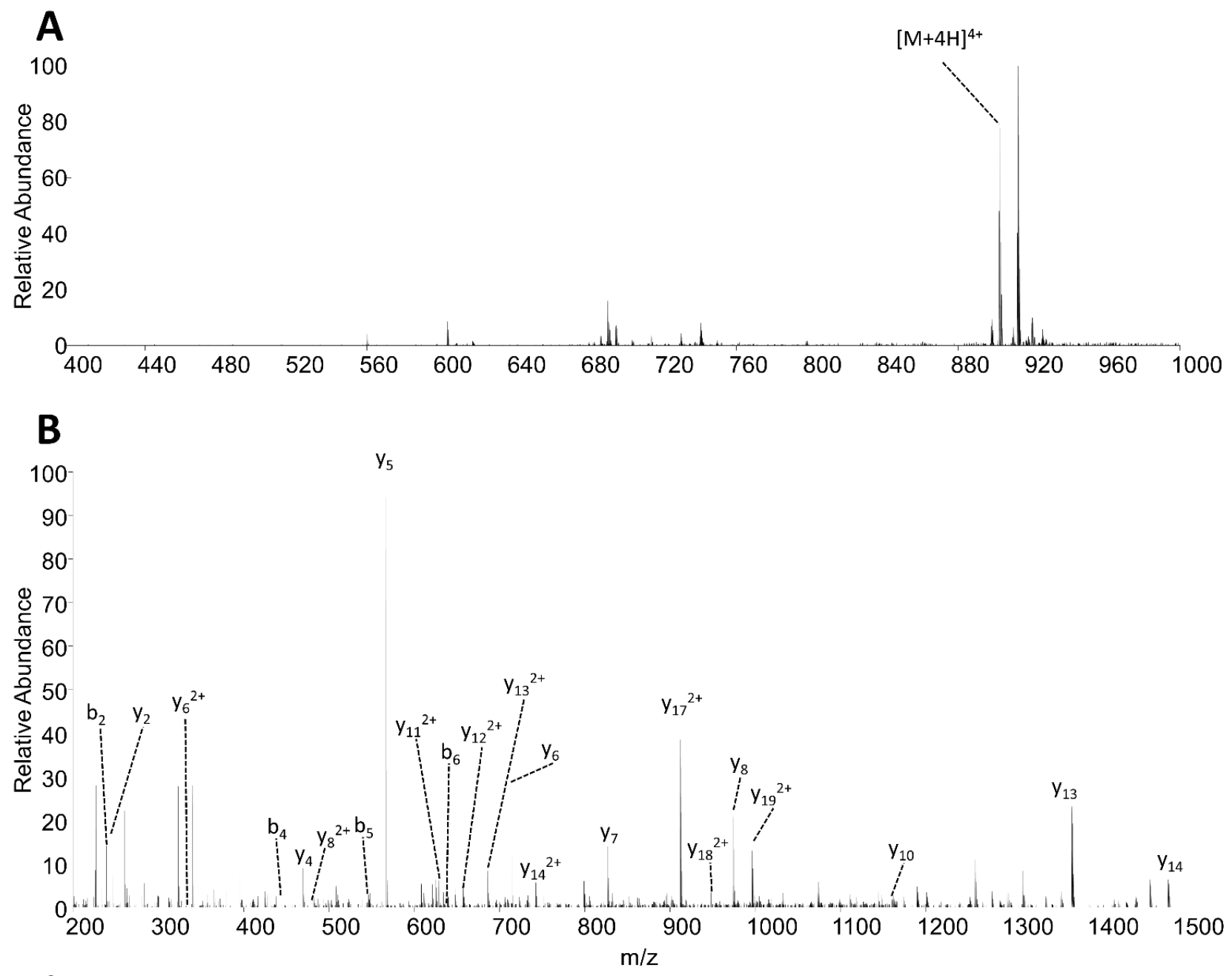

C

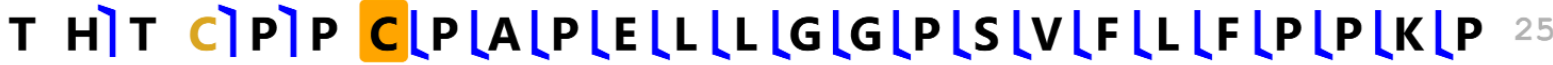

$26 \mathbf{K}$ 
Figure S5: (A) MS1 and (B) MS/MS spectra (HCD NCE 30, 3+ charge state) of one payload-containing peptide identified from the Byonic database search of the bottom-up dataset. The spectra are shown for a peptide which is believed to be a false match. (C) The resulting fragmentation map; no payloadcontaining product ions were identified. The cysteine highlighted in gold is presumed to contain the payload conjugation
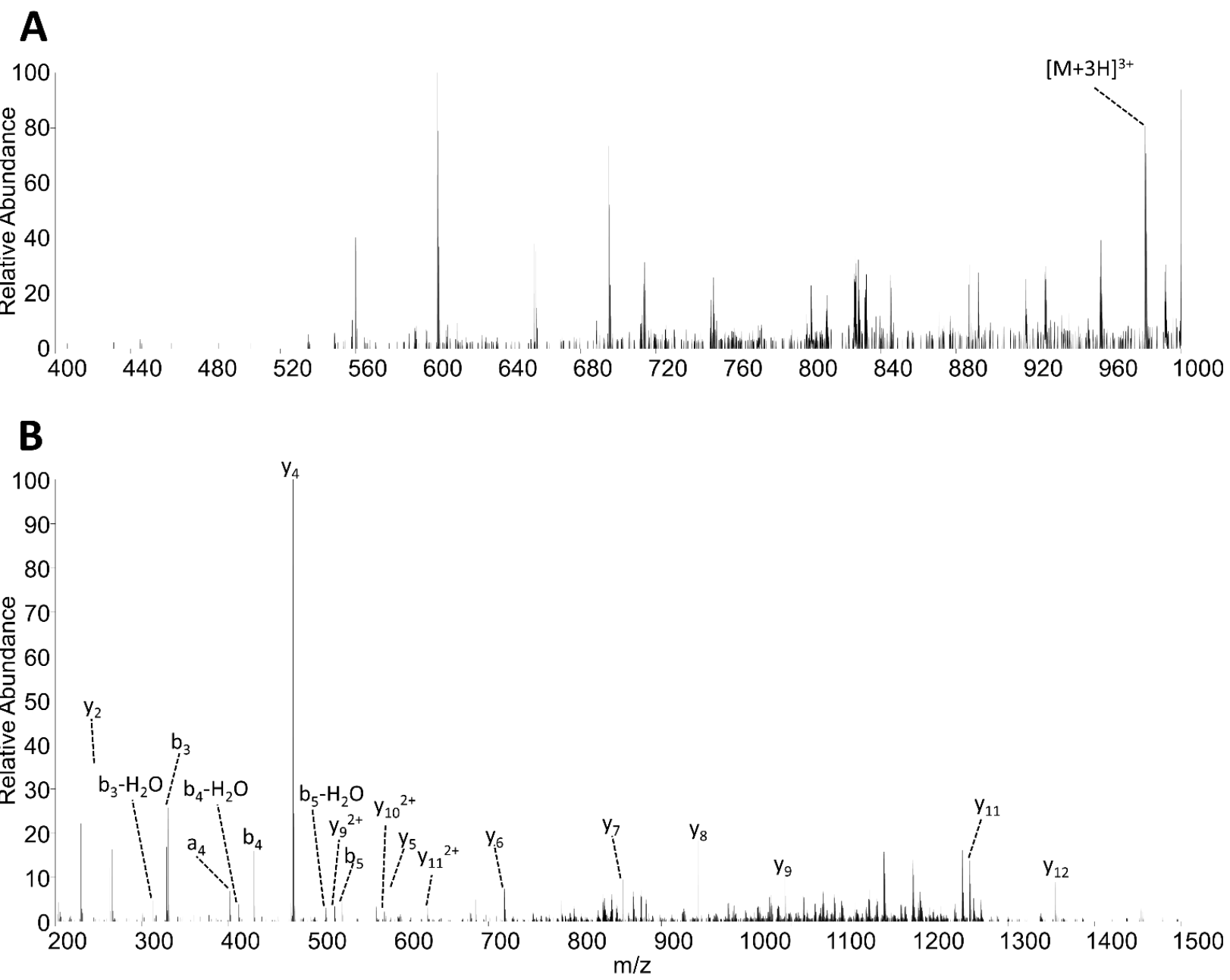

C

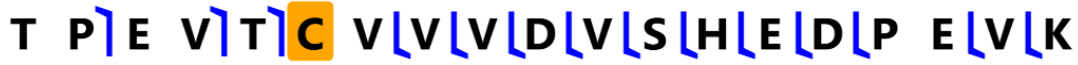


Figure S6: MS/MS spectra of the linker (1+ charge state). (A) UVPD (1 pulse, $1 \mathrm{~mJ})$, (B) HCD (10 NCE). (C) The structure of the linker is illustrated with proposed color-coded cleavages that lead to the observed fragment ions along with the corresponding neutral losses indicated in black. (D) The same cleavages are mapped onto the linker-with-payload structure to indicate several predicted fragmentation pathways and expected neutral losses that might contribute to fragment ions in the MS/MS spectra of ADCs.

A

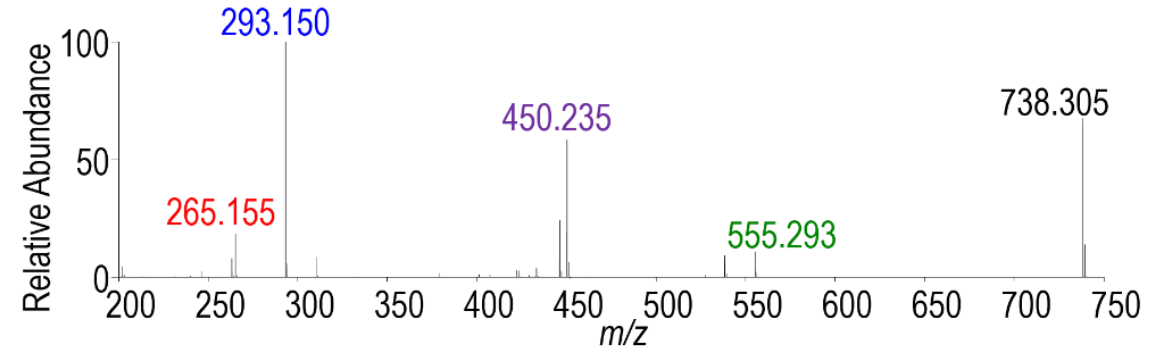

B
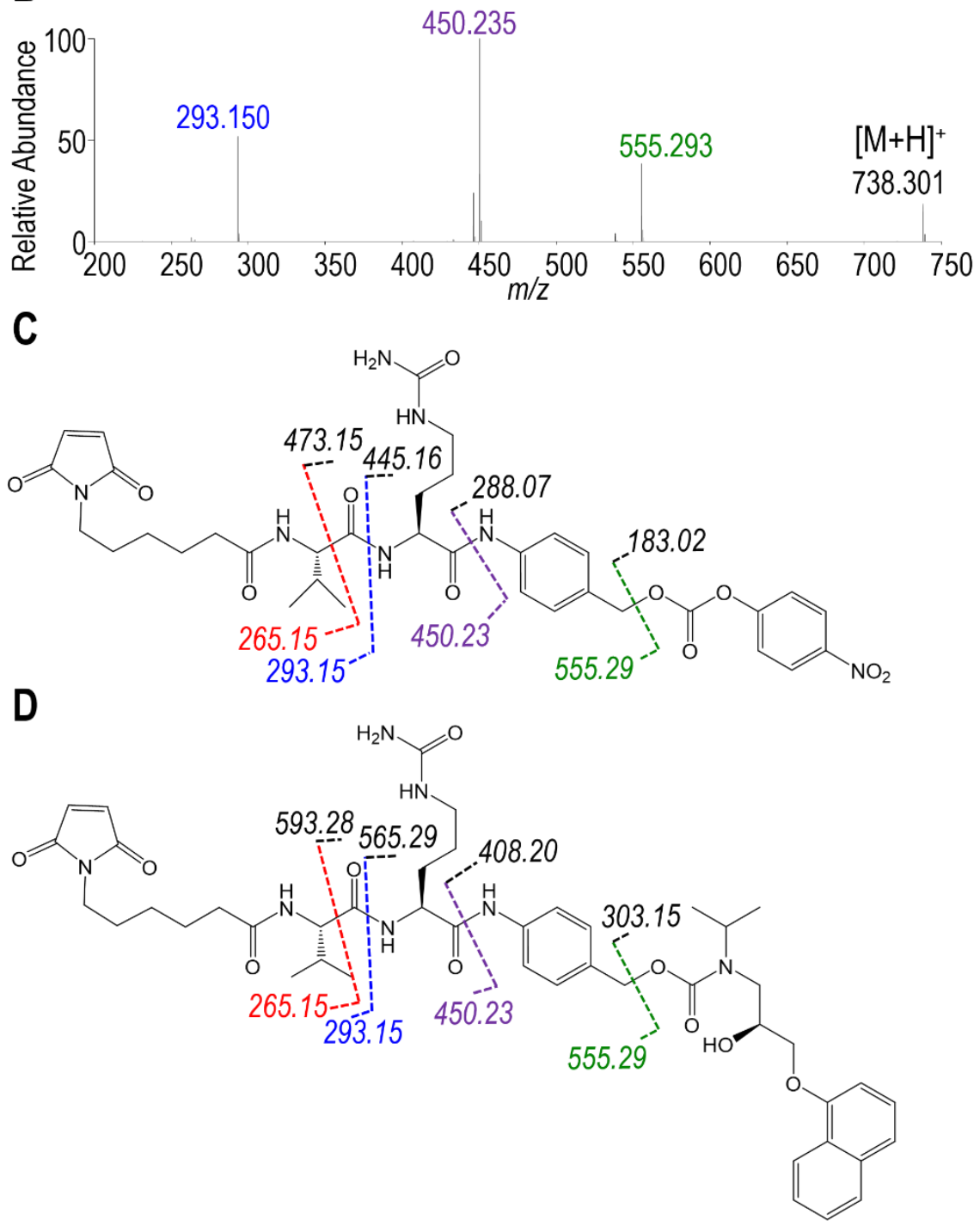
Figure S7: MS1 spectra for each of the seven corresponding LC peaks labeled in Figure 1. Subunits were identified from the deconvoluted mass, shown to the left of each spectrum. The subunits shown are: (A) $\mathrm{Fc} / 2$ (B) Lc, (C) Lc with one payload, (D) Fd', (E) Fd' with one payload, (F) Fd' with two payloads, and (G) $\mathrm{Fd}^{\prime}$ with three payloads. For each MS1 spectrum, the ADC scheme to the right highlights the segment of the antibody in green and display the number of payloads as pink circles.
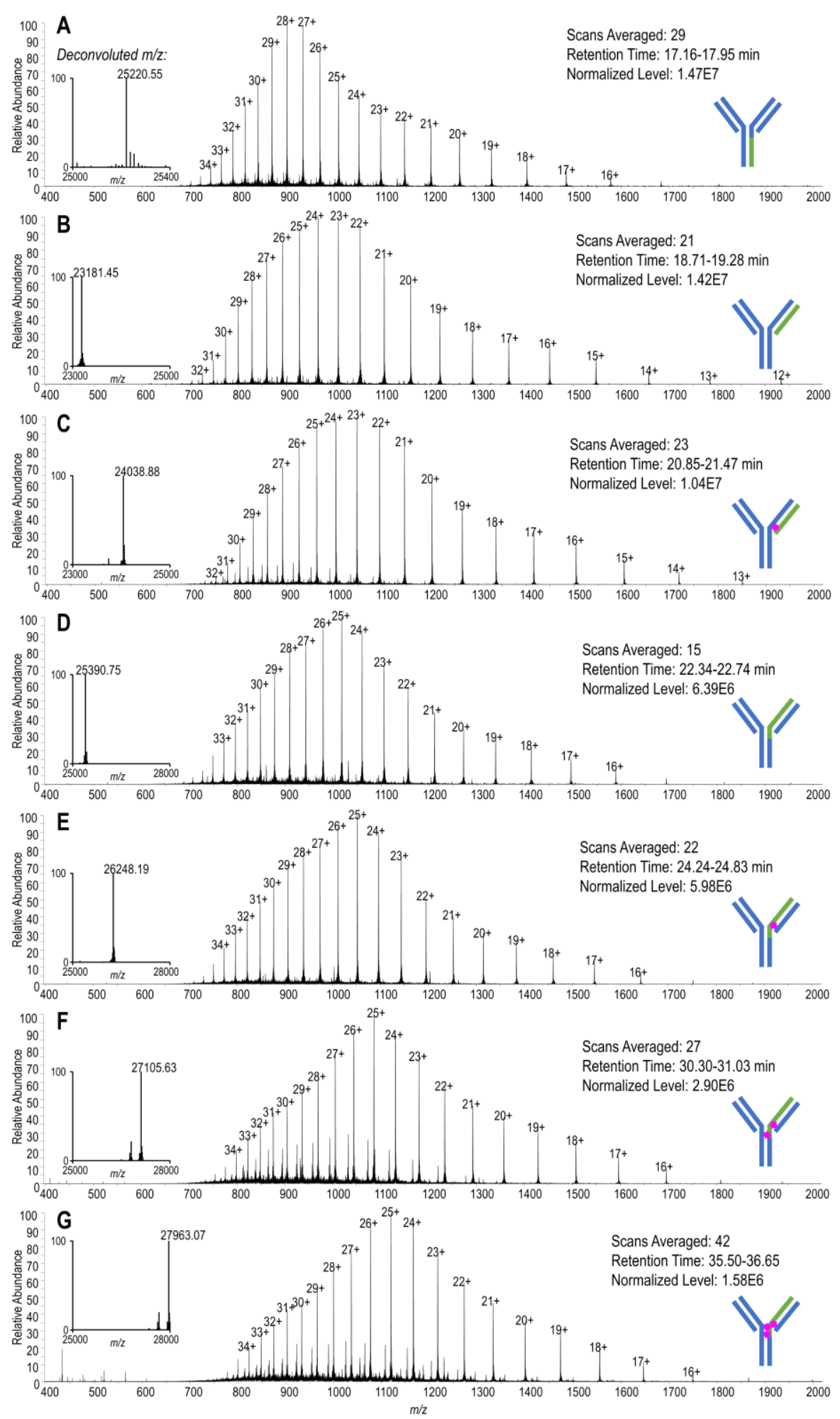
Figure S8: Product ion arrays showing sites of backbone cleavages for (A) Fd' and (B) Fd' with one payload on C224 based on the optimized activation conditions including ETD (15 ms), EThcD (10 ms with $10 \mathrm{NCE}$ ), and UVPD ( 1 pulse at $1 \mathrm{~mJ}$ ). The $25+$ charge state was activated.

A

N-Terminal C-Terminal

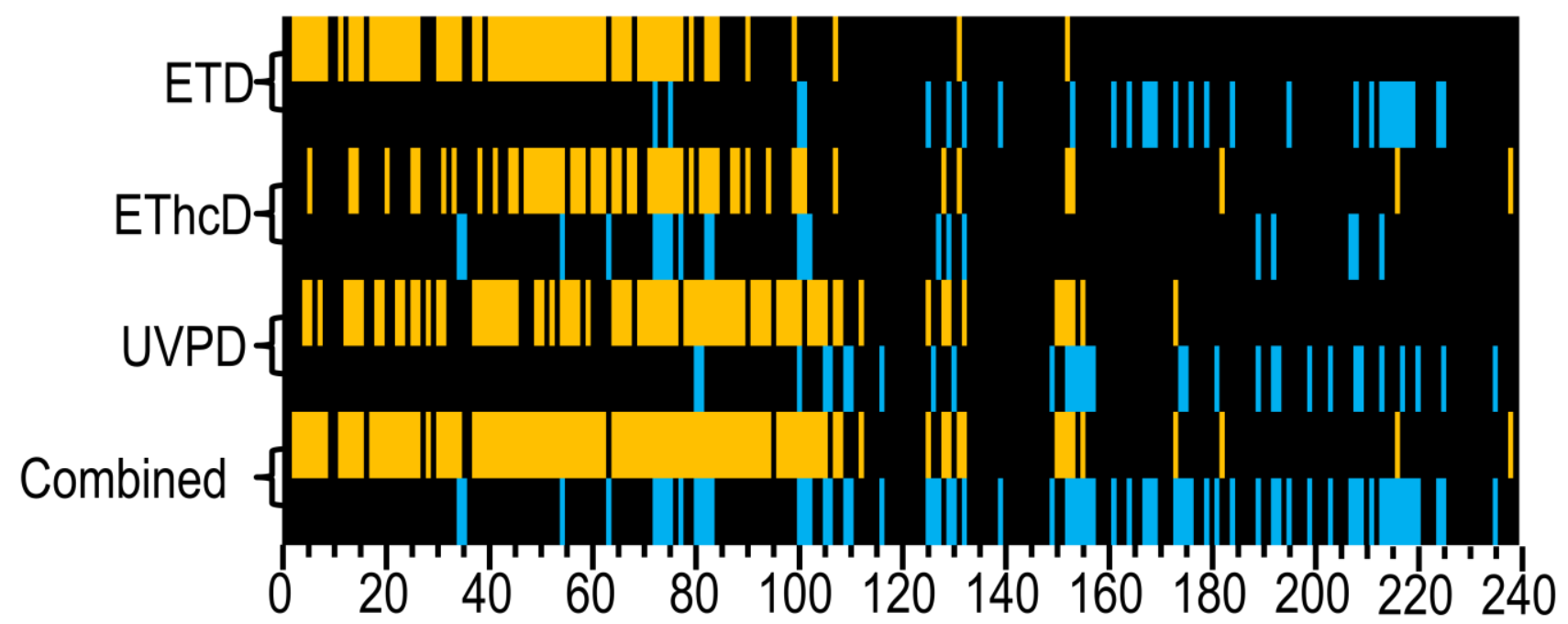

B

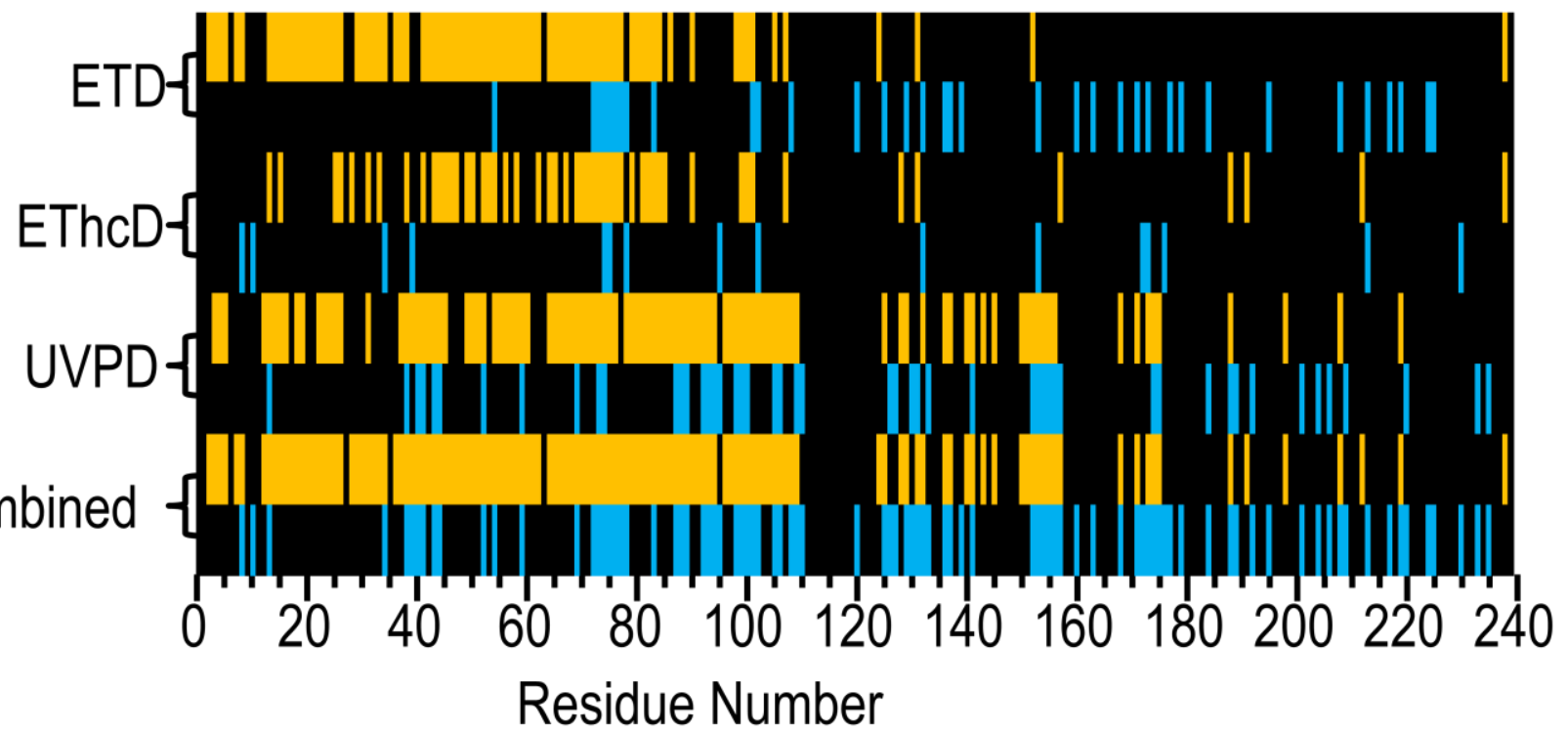


Figure S9: Product ion arrays showing sites of backbone cleavages for (A) Fd' with two payloads on C230 and C233 and (B) Fd' with three payload on C224, C230 and C233 based on the optimized activation conditions including ETD ( $15 \mathrm{~ms})$, EThcD (10 ms with $10 \mathrm{NCE})$, and UVPD ( 1 pulses at $1 \mathrm{~mJ})$. The 25+ charge state was activated.

A

N-Terminal C-Terminal

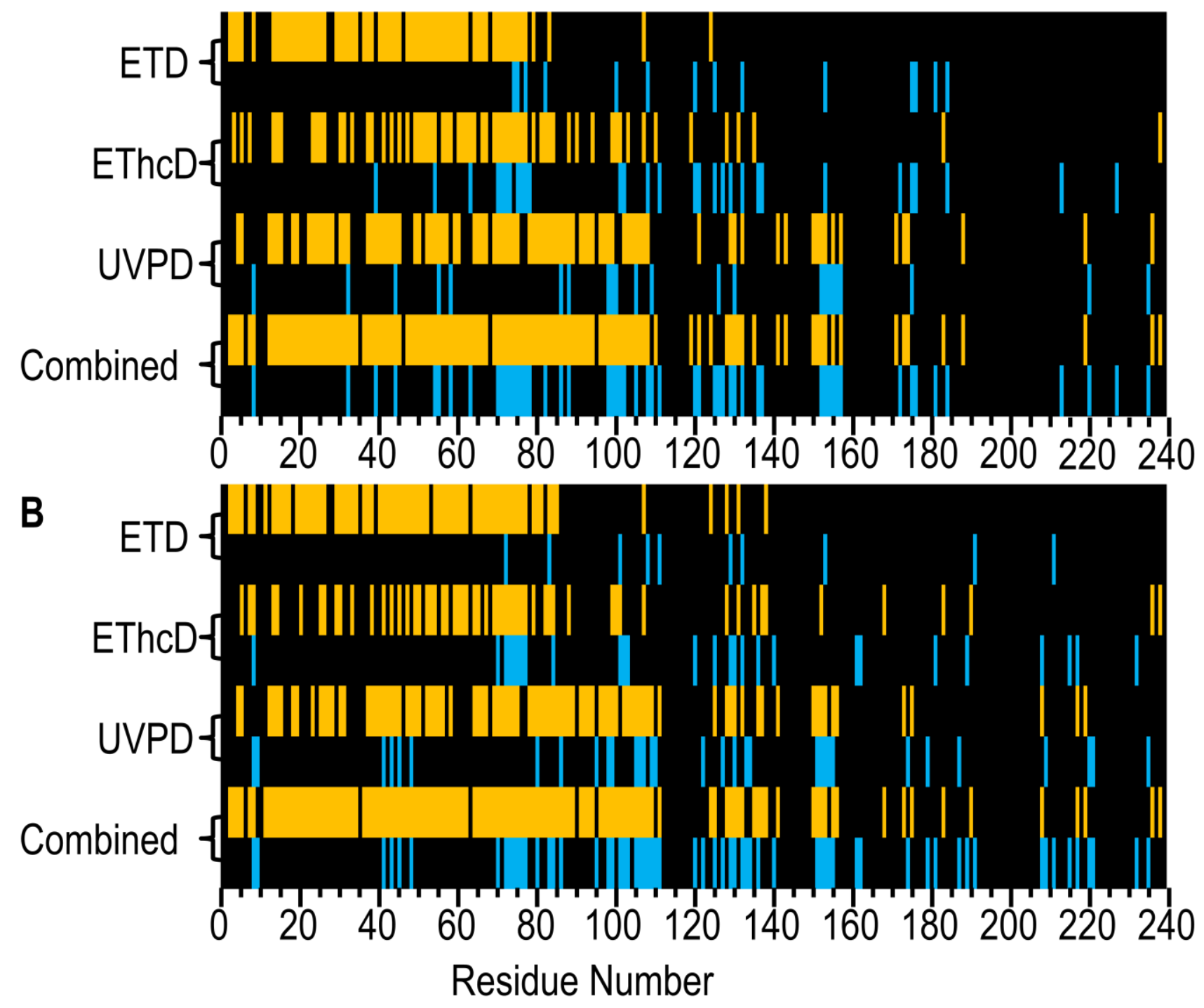


Figure S10: MS/MS data for EThcD (10 ms $10 \mathrm{NCE}$ ) of the 25+ charge state of Fd' with one payload. (A) Sequence coverage map with the payload positioned at C224. (B) Expansion of payload-free $\mathrm{y}_{10}{ }^{+}$which localizes the payload to C224 or C230. (C) Expansion of bis-payload-containing ion $z_{27}{ }^{3+}$. (D) Expansion of payload-free ion $\mathrm{C}_{213}{ }^{21+}$. The backbone cleavage sites that lead to the fragment ions that were selected for expansion are circled in purple on the sequence coverage map. CDRs are shaded in blue on the sequence coverage maps, and the payload conjugation site is highlighted in gold.

A

E V Q L L E S GLG DLL V R P]G G]S L R L S C A A S

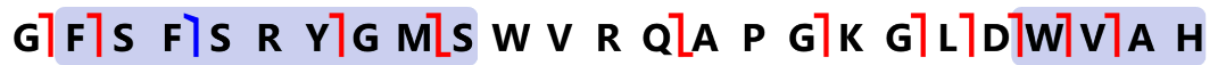

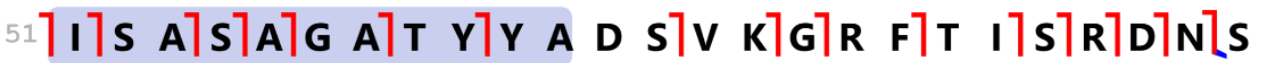

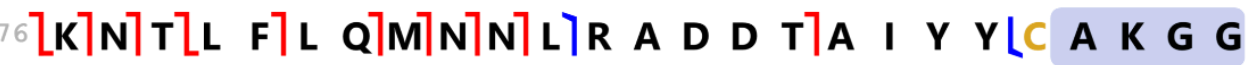
1017 K]Q]W L I P W FlD P W G Q G T L V T V S S A S T K 125 126 G P S VךF P LlA P S S K S T S G G T A A L G C L V 150 151 K D YLF P E P VlT V S W N S G A L T S G V HLTLF P 175 176 ALV L Q S S G L Y S L S S V\V T V]P S S S L G T Q 200 201 T Y I C N V N H K P S N th V D K V E P K S C D 225

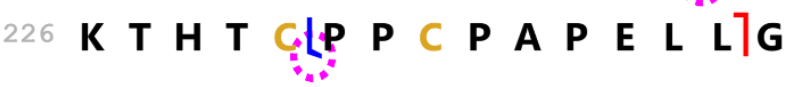
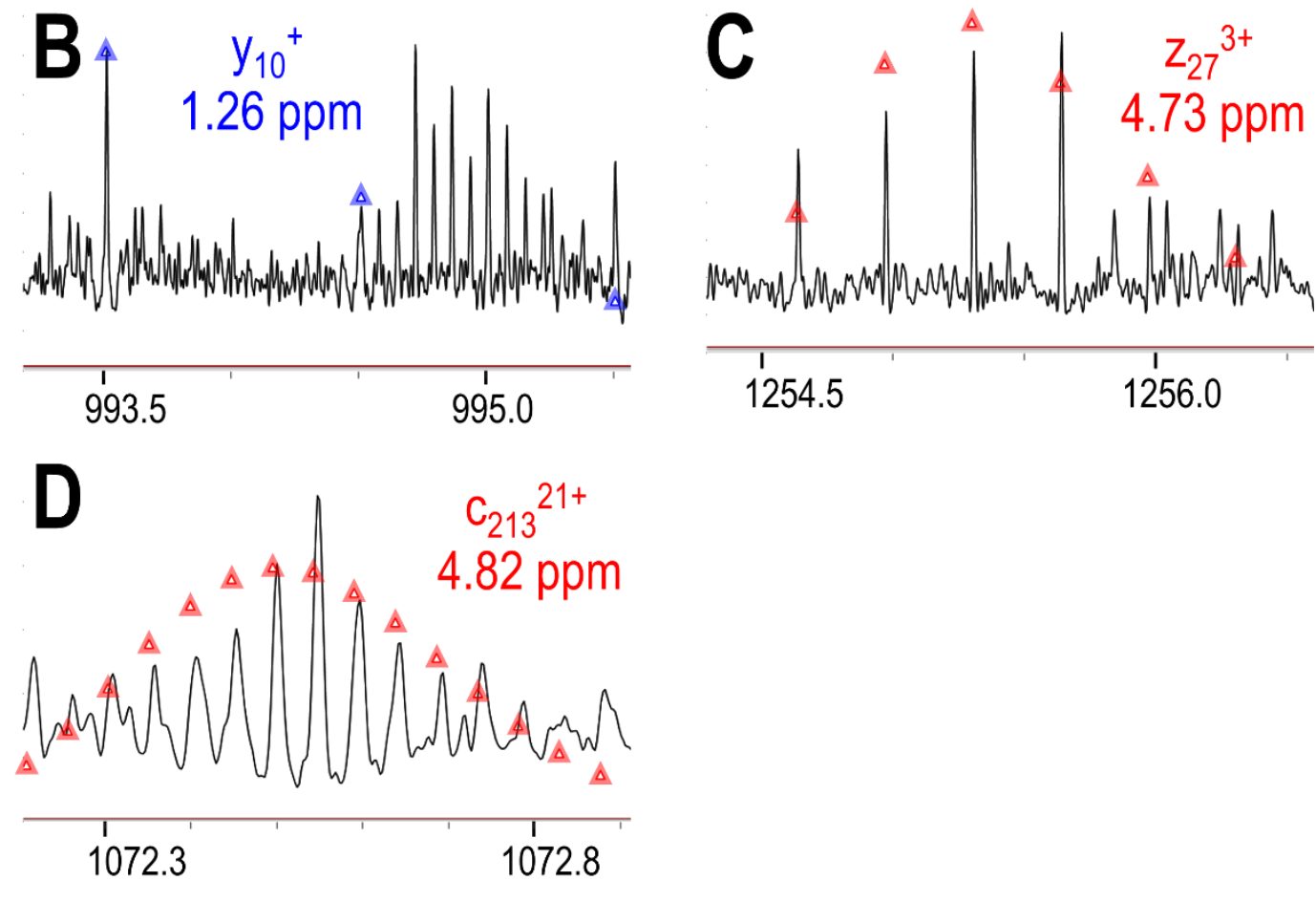

a b/y c/z fragment ion types 
Figure S11: MS/MS data for UVPD (1 pulse $1 \mathrm{~mJ}$ ) of the $25+$ charge state of Fd' with two payloads. (A) Sequence coverage map with the payloads positioned at C230 and C233. (B) Expansion of bis-payloadcontaining $\mathrm{y}_{20}{ }^{3+}$ (with one hydrogen loss). (C) Expansion of payload-free $\mathrm{a}_{174}{ }^{18+}$ (with one additional hydrogen). (D) Expansion of payload-free $\mathrm{a}_{220}{ }^{22+}$ (with one additional hydrogen). The backbone cleavage sites that lead to the fragment ions that were selected for expansion are circled in purple on the sequence coverage map. CDRs are shaded in blue on the sequence coverage maps, and the payload conjugation site is highlighted in gold.

A

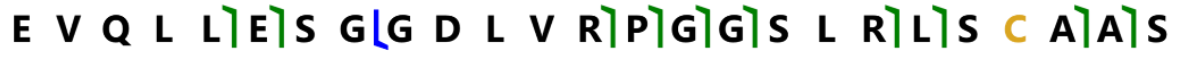

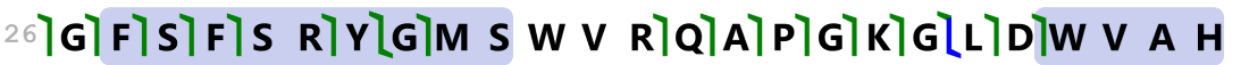

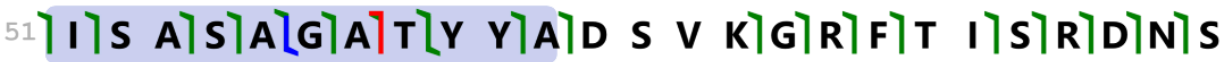

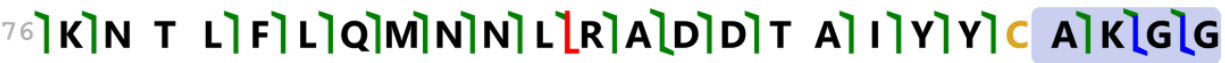

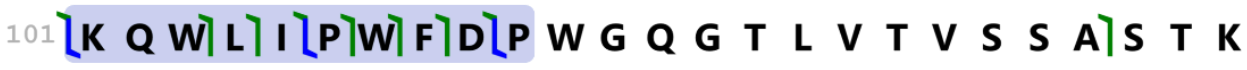
126 GLP S V FlP]L A]P S S K S T S G G]T A A L G C L V 150

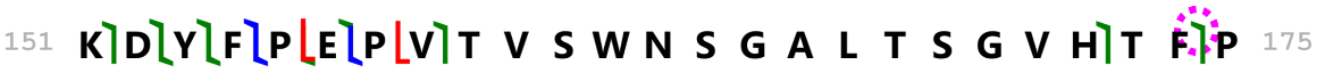
${ }_{176}$ A V L Q S S G L Y S L S S V\V T V P S S S L G T Q 200 201 T Y I C N V N H K P S N T K V D K K V 226 K T H T C P P C P ALP E]L L G
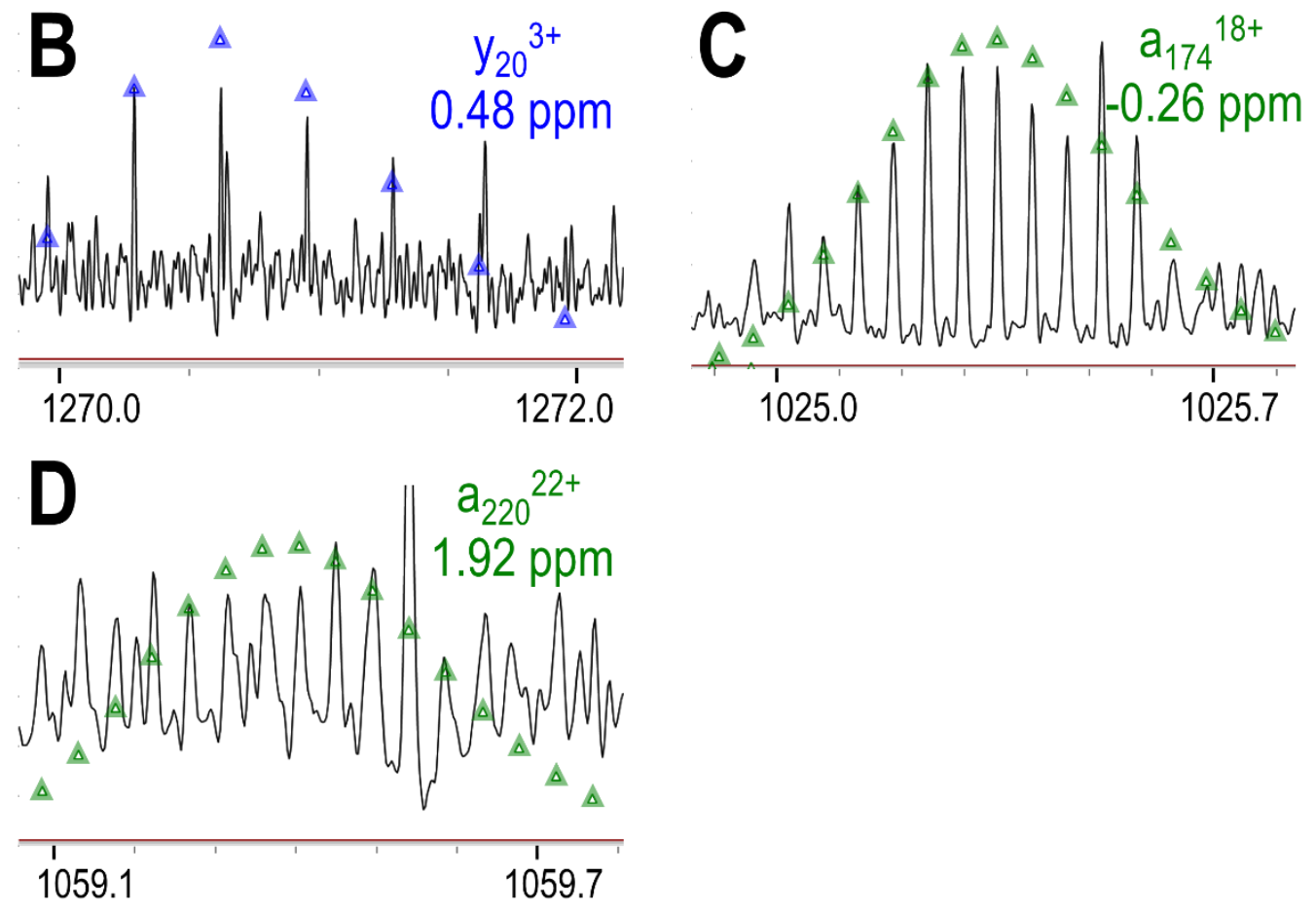

\section{$\square \mathrm{a} / \mathbf{x} \quad \mathrm{b} / \mathbf{y} \quad \mathrm{c} / \mathbf{z}$ fragment ion types}


Figure S12: Sequence coverage map for ETD $(15 \mathrm{~ms})$ of the 25+ charge state of Fd' with two payloads positioned at C230 and C233. CDRs are shaded in blue on the sequence coverage maps, and the payload conjugation site is highlighted in gold.

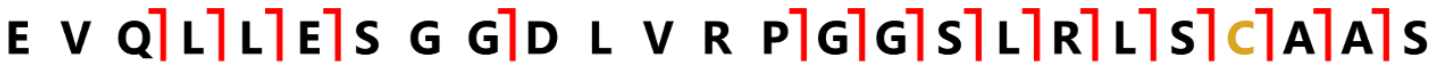

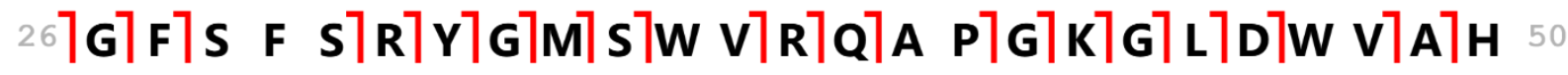

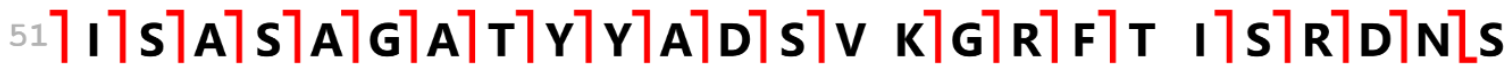
$\left.{ }^{76}[K\rceil N L T\right\rceil L$ FlL Q Q M N]N L R A D D T A I Y Y C A K G G
$101\lfloor$ K Q W L I P W FlD P W G Q G T L V T V SLS A S T K 125
126 G P S V F P LLA P S S K S T S G G T A A L G C L V 150
151 K D YLF P E P V T V S W N S G A L T S G V H T F P 175
${ }_{176}$ LALV L Q S SLG L YLS L S S V V T V P S S S L G T Q 200
201 T Y I C N V N H K P S N T K V D K K V E P K S C D 225
226 K T H T C P P C P A P E L L G 
Figure S13: MS/MS data for EThcD (10 ms $10 \mathrm{NCE}$ ) of the $25+$ charge state of Fd' with two payloads. (A) Sequence coverage map with the payloads positioned at C230 and C233. (B) Expansion of bis-payloadcontaining $\mathrm{Z}_{13}{ }^{2+}$ which localizes the payloads to $\mathrm{C} 230$ and C233. (C) Expansion of bis-payload-containing $\mathrm{z}_{27}{ }^{3+}$. The backbone cleavage sites that lead to the fragment ions that were selected for expansion are circled in purple on the sequence coverage map. CDRs are shaded in blue on the sequence coverage maps, and the payload conjugation site is highlighted in gold.

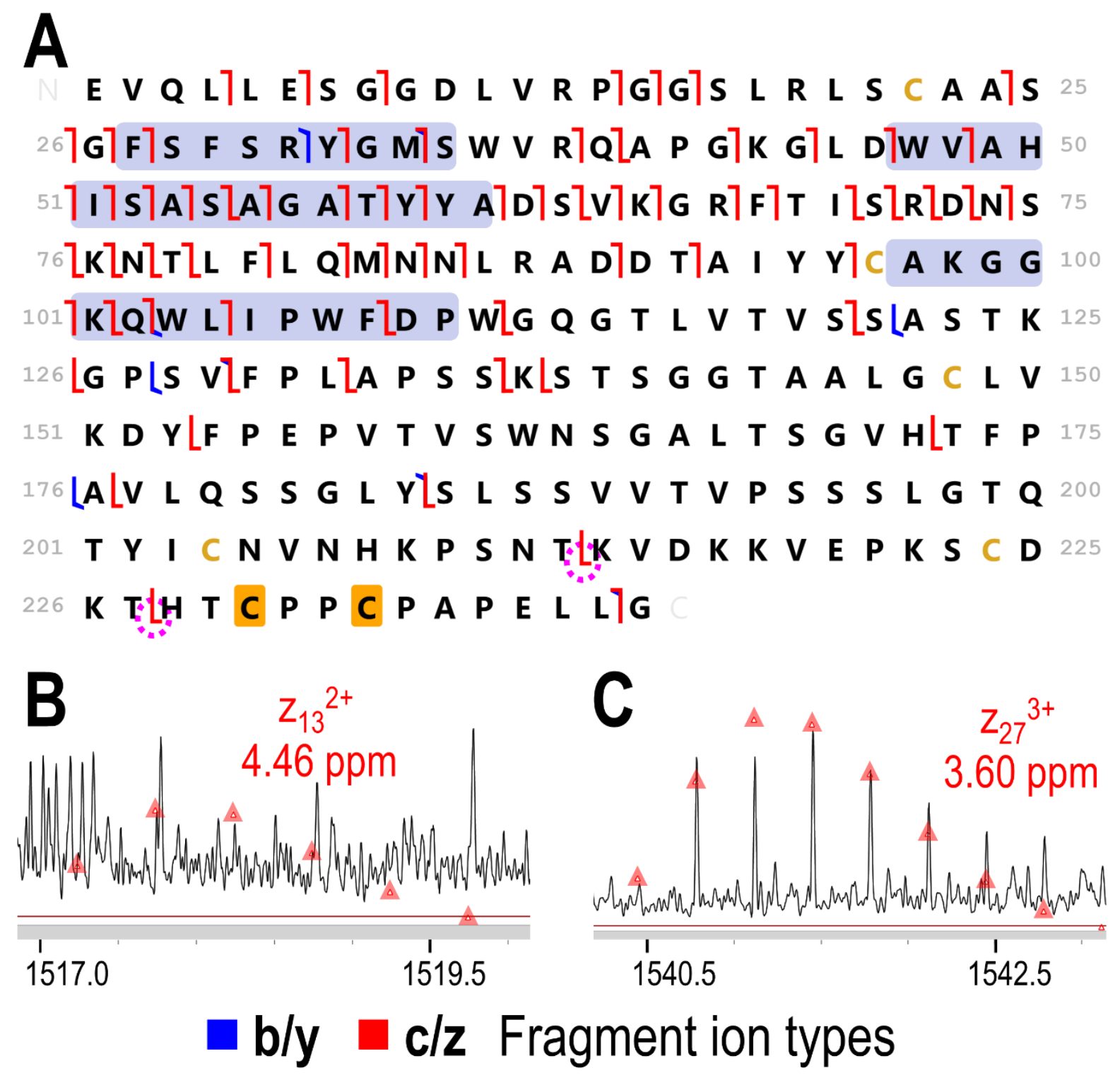


Figure S14: (A) Histogram displaying the number of backbone cleavage locations for Fd' with three payloads from UVPD (1 pulse, $1 \mathrm{~mJ})$, ETD (15ms), and EThcD ( $10 \mathrm{~ms}, 10 \mathrm{NCE})$ of the 25 + charge state, segregated into fragment ions that contain the payload (orange bars) and those that do not (blue bars). Sequence coverage maps are displayed for (B) UVPD, (C) ETD and (D) EThcD. CDRs are shaded in blue on the sequence coverage maps, and the payload conjugation sites $(C 224, C 230, C 233)$ are highlighted in gold.

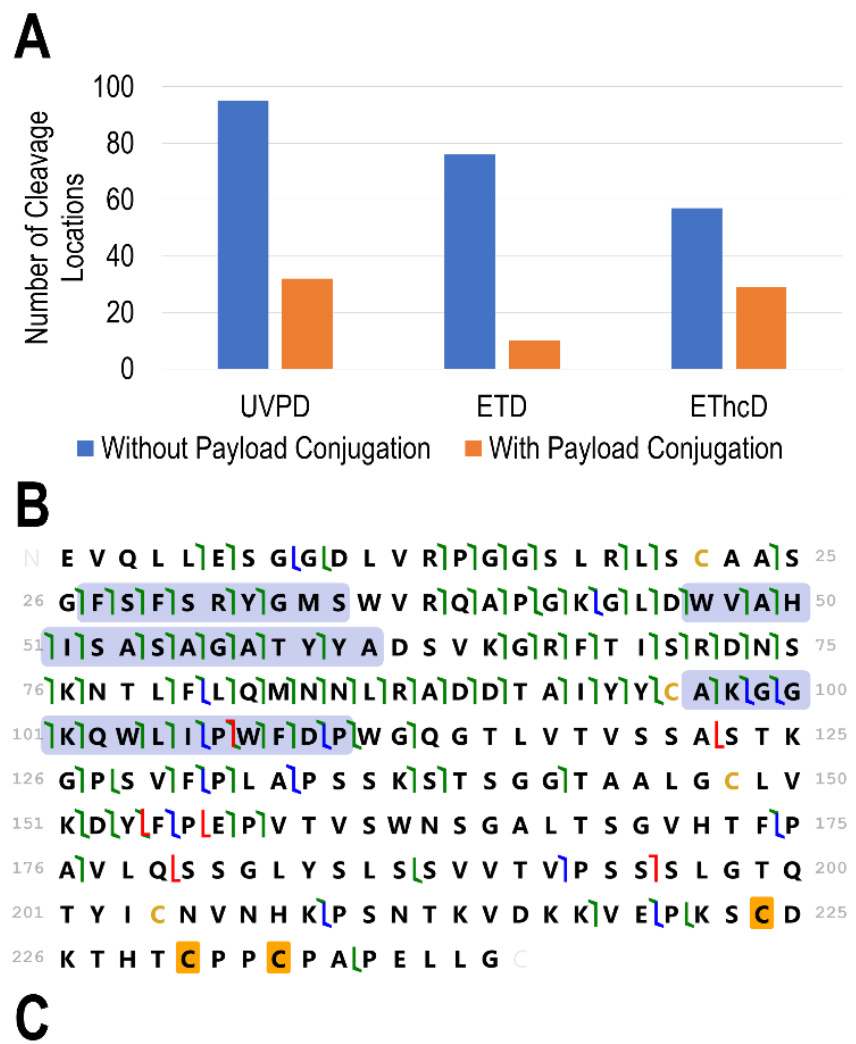

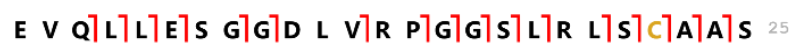

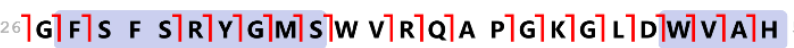

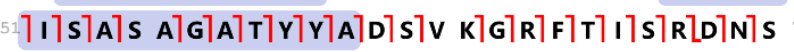
$\left.\left.\left.{ }^{6}\right\rceil K \backslash N\right\rceil T\right\rceil L$ F $\left.\left.\left.\left.\rceil L\right\rceil Q\right\rceil M\lfloor N\rceil N\right\rceil L\right\rceil R$ A D D T A I Y Y C A K G G KLQW L I PW FlD P WLG Q G T L V T V S S A S T K

${ }^{126} 7 \mathbf{G}$ P S VlF P LlA P S S K S T]S G G T A A L G C L V $1:$ ${ }_{151}$ K D YLF P E P V T V SWN S GAL T S G VH T F P

${ }_{176}$ A V L Q S S G L Y S L S S V V TLVPS S S L G T Q 2

201 T Y I C N V N H K P S LN T K V D K K V E P K S C D

226 K T H T C P P C P A P E L L G

D E V Q L L EךS G]G]D L V R P\G]G S L R L S TC A A S

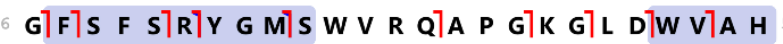

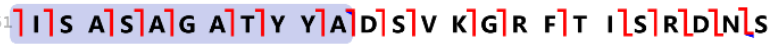

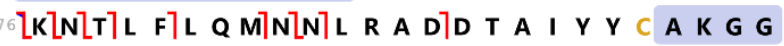
$0.7 K \backslash Q\rceil W L L$ I PW F\D PWG Q G T L V T V SLS A S T K ${ }_{126}[\mathbf{G}$ P S VlF $[\mathbf{P}$ LlA P S SlK S\T]S $\lfloor\mathbf{G}$ G T A A L G C L V 150 151 K D Y † P P P V T V S $\lfloor W\lfloor$ N S G A L T S $\rceil$ G V H T F P 175 ${ }_{176}$ A V L Q S SLG L Y)S L S S VLV T)V P S S S L G T Q 200 201 T Y I C N V N HLK P S N T K VLD KLK V E P K S C D 225 226 K T H T C P P[C P A P E $]$ L L $]$ G 
Figure S15: Percent contributions for different peaks of $(A) L C$ and $(B) F^{\prime} d^{\prime}$ based on areas of the extracted ion chromatograms. For (A) LC $(26+, 25+, 24+, 23+$, and $22+$ charge states are all included) and for (B) Fd' $\left(28+, 27+, 26+, 25+, 24+\right.$, and $23+$ charge states are all included). The $\mathrm{Fd}^{\prime}+1$ payload and $\mathrm{Fd}^{\prime}+2$ payload species are each split into two peaks (labelled (a) and (b)). The dotted blue lines indicate the boundaries of the areas utilized for each contribution. (C) Summary of the possible ways to add together the peaks in part B to estimate the percentage of C224 containing a payload. (D) The final extracted ion chromatogram displays the expected conjugation sites for each peak associated with the Fd' subunit.

A

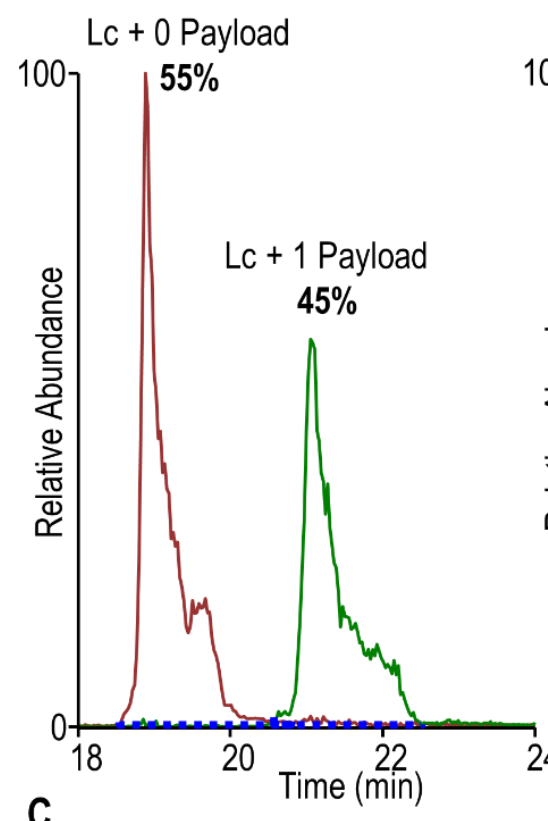

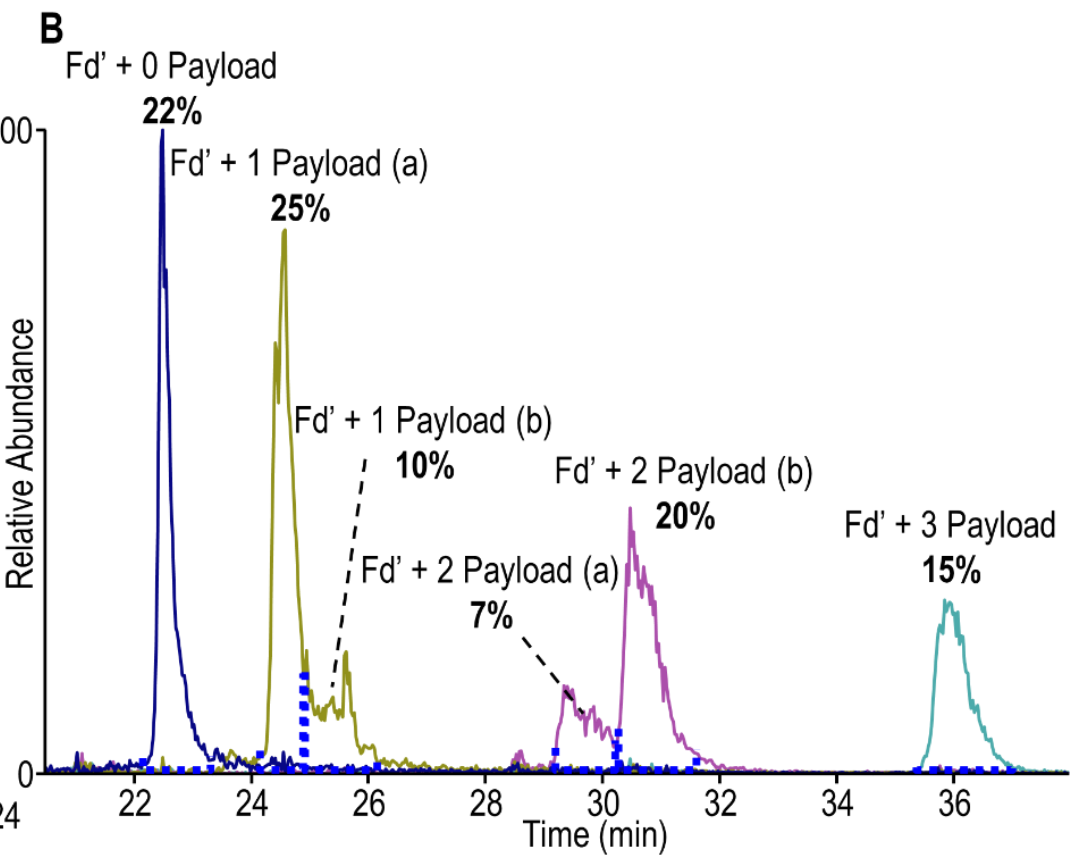

\section{Peaks added together}

\section{Percent of C224 Containing a Payload}

\begin{tabular}{ll}
\hline Fd' + 1 Payload (a), Fd' + 2 Payload (b), Fd' + 3 Payload & $60 \%$ \\
\hline Fd' + 1 Payload (a), Fd' + 2 Payload (a), Fd' + 3 Payload & $47 \%$ \\
\hline Fd' + 1 Payload (b), Fd' + 2 Payload (b), Fd' + 3 Payload & $45 \%$ \\
\hline Fd' + 1 Payload (b), Fd' + 2 Payload (a), Fd' + 3 Payload & $32 \%$
\end{tabular}

D

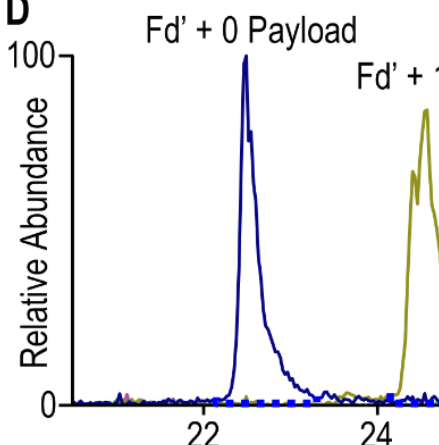

22

24

1 Payload (a) C224

Fd' + 1 Payload (b) $\quad$ Fd' +2 Payload (b) C230 or C233 $\quad$ C230 and C233

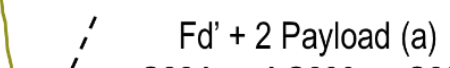

Fd' + 3 Payload

C224, C230, and C233 
Table S1. Excel spreadsheet includes lists of fragment ions identified and manually confirmed using TDValidator. The spreadsheets are segregated by subunit and include data for Lc with zero or one payload, and Fd' with zero, one, two or three payloads. Data for UVPD, (1 pulse $1 \mathrm{~mJ})$ ETD, (15 ms) and EThcD (10 ms $10 \mathrm{NCE}$ ) are included together on each spreadsheet. For each fragment ion listed, the fragmentation type is listed along with the theoretical mass, ppm error, charge state, monoisotopic $m / z$, signal-to-noise, abundance and score.

(1) Fornelli, L.; Ayoub, D.; Aizikov, K.; Beck, A.; Tsybin, Y. O. Middle-Down Analysis of Monoclonal Antibodies with Electron Transfer Dissociation Orbitrap Fourier Transform Mass Spectrometry. Anal. Chem. 2014, 86, 3005-3012.

(2) Fornelli, L.; Srzentić, K.; Huguet, R.; Mullen, C.; Sharma, S.; Zabrouskov, V.; Fellers, R. T.; Durbin, K. R.; Compton, P. D.; Kelleher, N. L. Accurate Sequence Analysis of a Monoclonal Antibody by TopDown and Middle-Down Orbitrap Mass Spectrometry Applying Multiple Ion Activation Techniques. Anal. Chem. 2018, 90, 8421-8429.

(3) Shaw, J. B.; Li, W.; Holden, D. D.; Zhang, Y.; Griep-Raming, J.; Fellers, R. T.; Early, B. P.; Thomas, P. M.; Kelleher, N. L.; Brodbelt, J. S. Complete Protein Characterization Using Top-Down Mass Spectrometry and Ultraviolet Photodissociation. J. Am. Chem. Soc. 2013, 135, 12646-12651.

(4) Holden, D. D.; McGee, W. M.; Brodbelt, J. S. Integration of Ultraviolet Photodissociation with Proton Transfer Reactions and Ion Parking for Analysis of Intact Proteins. Anal. Chem. 2016, 88, 1008-1016.

(5) Liu, J.; McLuckey, S. A. Electron Transfer Dissociation: Effects of Cation Charge State on Product Partitioning in Ion/Ion Electron Transfer to Multiply Protonated Polypeptides. Int. J. Mass Spectrom. 2012, 330-332, 174-181.

(6) Good, D. M.; Wirtala, M.; McAlister, G. C.; Coon, J. J. Performance Characteristics of Electron Transfer Dissociation Mass Spectrometry. Mol. Cell. Proteom. 2007, 6, 1942-1951. 